\title{
Paediatricians' knowledge of cardiac arrest guidelines
}

\author{
P W Buss, R J Evans, G McCarthy, T Scorrer, V Kumar
}

\begin{abstract}
A telephone questionnaire was undertaken on middle grade trainee paediatricians to test their knowledge of European Resuscitation Council guidelines. Fifty seven responded of whom only $15(26 \%)$ offered a correct sequence of management for asystole and eight (14\%) failed to identify adrenaline in their management. For ventricular fibrillation only 18/57 (32\%) identified a correct sequence and very poor specific knowledge was identified.

Paediatricians will under perform in the event of cardiac arrest in children without improved training in resuscitation.

(Arch Dis Child 1996; 74: 47-49)
\end{abstract}

Keywords: cardiac arrest, resuscitation, guidelines.

It is fortunate that in most paediatric units cardiac arrest calls are uncommon. A high level of skill and knowledge is required in circumstances which represent a most stressful clinical situation. The difficulties encountered are due to the low frequency of paediatric cardiac arrest in comparison with adults, ${ }^{1}$ physical problems in establishing basic life support in a small patient, and technical difficulties in securing the airway and attaining vascular access.

Guidelines for paediatric resuscitation published by the European Resuscitation Council $(E R C)^{2}$ are incorporated within the advanced paediatric life support (APLS) protocols ${ }^{3}$ for cardiac arrest. We attempted to identify whether middle grade trainee paediatricians in England and Wales had competent knowledge of these protocols for the management of children with cardiac arrest from asystole or ventricular fibrillation given that they would be likely to be leading resuscitation efforts.

Department of
Paediatrics, Royal
Gwent Hospital,
Cardiff Road, Newport,
Gwent NP9 2UB
P W Buss
G McCarthy
T Scorrer
V Kumar

Department of Emergency Medicine, Cardiff Royal

Infirmary

R J Evans

Correspondence to: Dr Buss.

Accepted 5 September 1995

\section{Methods}

Sixty eight hospitals (including 10 teaching hospitals) in 12 health regions were identified from The Medical Directory $1993^{4}$ as units accepting acute paediatric admissions (the local switchboard operator confirmed resident, middle grade paediatric cover). A single paediatrician was contacted in each unit during evening hours to reduce conference between colleagues (and also to reduce the costs of the study).

A questionnaire (appendix) was designed initially to test knowledge of the APLS/ERC sequences and then specific details of the resuscitation. Questions were posed in a predefined sequence. Hypothetical cases of asystole and ventricular fibrillation (confirmed by an electrocardiogram (ECG)) were posed. It was stressed that good basic life support and vascular access was maintained throughout. We included general questions about the paediatricians' qualifications and whether there had been in-house training or APLS course attendance. We also included a question related to the route of administration of drugs if no peripheral vascular access had been obtained. Analysis of the results was undertaken by PWB using an answer template.

\section{Results (table)}

Fifty seven resident middle grade paediatricians responded and completed the questionnaire.

\section{ASYSTOLE}

(A) Sequence and management

Fifteen paediatricians $(26 \%)$ related a sequence in keeping with APLS/ERC resuscitation guidelines. Forty two (74\%) gave incorrect answers. (Four excluded only fluid from the sequence, one of whom additionally incorporated calcium gluconate in the sequence.)

Eight $(14 \%)$ failed to identify adrenaline as part of the protocol for paediatric asystole. Of the remaining $49,19(41 \%)$ failed to recognise the need for repeated adrenaline dosages or incorrectly included the second dosage after administration of direct current (DC) shock or calcium gluconate infusion. Thirty five (61\%) did not mention fluid as an essential part of resuscitation in paediatric asystole and 35 (61\%) gave no concept of cycles of cardiopulmonary resuscitation and drug administration. In addition $34(60 \%)$ did not include the use of alkali but we recognise this as debatable so did not include it as a 'fail' in the sequence answer template.

\section{(B) Specific answers}

Of those with incorrect sequence answers, 25/42 (60\%) possessed full MRCP (UK), 39 (90\%) had not received APLS training, and 29 $(70 \%)$ had received no in-house training. Twenty one (36\%) had incorrect knowledge of the initial adrenaline dosage and $40(70 \%)$ expressed either no requirement for fluid or an inadequate volume of fluid replacement. Forty nine $(86 \%)$ recognised adrenaline as the drug used in asystole but $11(22 \%)$ failed to increase subsequent adrenaline dosage. Forty $(70 \%)$ either had no concept of cycles (35) or a cycle time in excess of one minute longer than recommended (5). The longest time between adrenaline dosages was recorded as $\mathbf{2 0}$ minutes. 
Summary of responses to general and specific questions on the management of paediatric cardiac asystole and ventricular fibrillation

\begin{tabular}{llll}
\hline Asystole & No (\%) response & Ventricular fibrillation & No (\%) response \\
\hline Sequence correct & $15 / 57(26)$ & Sequence correct & $18 / 57(32)$ \\
Specific questions & $36 / 57(63)$ & Shock energy 1 correct & $20 / 57(35)$ \\
$\quad$ Adrenaline 1 correct & $17 / 57(29)$ & Adrenaline 1 correct & $21 / 57(37)$ \\
Fluid (mentioned) & $38 / 57(57)$ & Shock energy 2 correct & $20 / 57(35)$ \\
Adrenaline 2 correct & $17 / 57(30)$ & Adrenaline 2 correct & $16 / 57(28)$ \\
Cycles (CPR /adrenaline) & & Cycles (shock/adrenaline) & $17 / 57(30)$ \\
Additional points & & Atropine included & $1 / 57(2)$ \\
Atropine included & $16 / 57(28)$ & Calcium included & $2 / 57(4)$ \\
Calcium included & $9 / 57(16)$ & Lignocaine included & $18 / 57(32)$ \\
DC shock included & $15 / 57(26)$ & DC shock omitted & $6 / 57(10)$ \\
Adrenaline omitted & $8 / 57(14)$ & & \\
\hline
\end{tabular}

${ }^{\star} \mathrm{CPR}=$ cardiopulmonary resuscusitation.

Sixteen $(28 \%)$ of 57 middle grade trainee paediatricians included atropine within their asystole protocol; nine included calcium gluconate. Fifteen (26\%) included DC shock in their management of asystole. Eleven of 42 $(26 \%)$ were wrong with regard to all five specific elements.

\section{VENTRICULAR FIBRILLATION}

\section{(A) Sequence and management}

Eighteen of $57(32 \%)$ related a correct sequence. Ten of this group had similarly correct responses for asystole. Six $(10 \%)$ claimed to have 'no idea' of resuscitation of ventricular fibrillation and failed to include DC shock in their response. Thirty two (56\%) recognised adrenaline for use in the ventricular fibrillation sequence but only $24(42 \%)$ recognised the concept of cycles of shock/adrenaline with cardiopulmonary resuscitation. Thirty nine $(68 \%)$ gave an incorrect sequence, 26 six of whom $(68 \%)$ possessed full MRCP (from a total of 34). Six of the 10 who had attended an APLS course were incorrect while $26(66 \%)$ of those who answered incorrectly claimed to have had no in-house training on resuscitation for ventricular fibrillation.

\section{(B) Specific answers}

Thirty seven $(65 \%)$ gave no value or incorrect values for initial DC shock energy. Thirty six $(63 \%)$ gave a wrong initial dosage of adrenaline. Thirty seven $(65 \%)$ of those questioned failed to identify correct subsequent shock energy and $41(72 \%)$ did not recognise the need for additional doses of adrenaline or gave incorrect dosage. Forty (70\%) had no concept of cycles of cardiopulmonary resuscitation and treatment (33/40), or gave an incorrect cycle length $(7 / 40)$ - the longest time identified between shocks was 15 minutes.

Two respondents included calcium gluconate in their sequence and one included atropine sulphate. One identified amiodarone and one bretylium. Lignocaine was identified as an essential part of the sequence by 18 . Twenty four of the 39 who were incorrect $(62 \%)$ had all five elements incorrect.

Fifty two $(91 \%)$ of those questioned identified the intraosseous route as an acceptable route should peripheral venous access be unobtainable in the first 90 seconds. All except one identified the endotracheal route as a route of drug administration. Two identified the intracardiac route as the preferred route for drug administration should an intraosseous route be unavailable.

\section{Discussion}

The results of this survey should concern paediatricians throughout England and Wales. The APLS/ERC protocols are not difficult to assimilate - containing relatively brief information. The results of our survey are similar to the findings of Tham et al. ${ }^{5}$ Two thirds of middle grade trainee paediatricians (often identified by colleagues as the leader of the resuscitation team) were unable to identify the correct sequence of resuscitation in two common arrhythmias encountered in childhood cardiac arrest. Very poor specific knowledge was recorded. Eight paediatricians failed to include adrenaline in their management of asystole and six failed to recognise DC shock in management of ventricular fibrillation. Atropine was mentioned as useful for asystole by over one quarter of respondents. The ERC guidelines clearly indicate that atropine has few specific uses in resuscitation and is not necessary in asystolic cardiac arrest. ${ }^{2}$ Calcium is associated with reperfusion injury and is no longer recommended in cardiac arrest, yet nearly a fifth of respondents would have used it. Lignocaine was included early in the ventricular fibrillation sequence by over a third of paediatricians. It is generally recognised that it can prevent ventricular fibrillation but it may not reverse the arrhythmia. A high percentage recognised the intraosseous route for achieving vascular access - significantly more than in a similar survey of more junior paediatricians in $1992 .^{6}$

Holders of the MRCP fared no better than their colleagues without the qualification. (Those without the MRCP frequently related that they were preparing for the examination.) We believe the paediatric MRCP examination should include compulsory sections on basic and advanced life support. The percentage of paediatricians with APLS certification giving incorrect answers was not surprising in view of studies indicating a need for repeated updating of basic life support knowledge and practical skills. Seventy one per cent of those questioned had received no in-house training on paediatric resuscitation - this is the obvious setting for regular updating on cardiopulmonary resuscitation.

Middle grade trainee paediatricians are often encouraged by other professional colleagues to be team leaders in paediatric resuscitation events. This study indicates that many are not equipped with adequate knowledge so are likely to under perform in resuscitation events. National standardisation of paediatric resuscitation is required and paediatric resuscitation skill attainment should be monitored locally and nationally. We believe that it is only through monitoring that adequate standards can be achieved and maintained, so optimising the likelihood of intact survival from cardiac arrest. 
1 Innes PA, Summers CA, Boyd IM, Molyneux EM. Audit of paediatric cardiopulmonary resuscitation. Arch Dis Child 1993; 68: 487-92.

2 Zidemann D. Working Party of the European Resuscitation Council: guidelines for paediatric life support. $B M \mathcal{F} 1994$; 308: $1349-55$.

3 Advanced Life Support Group. Advanced paediatric life support: the practical approach. London: BMJ Publishing support: the prac

4 Longman Information and Reference Division. The medical directory 1993. 149th Ed. London: Longman Group UK, 1993.

1993.

Tham KY, Evans RJ, Rubython ES, Kinnaird L. 1994; 309: 1408-9.

6 Buss PW, McCabe M, Evans RJ, Davies A, Jenkins H. A survey of basic resuscitation knowledge among resident paediatricians. Arch Dis Child 1993; 68: 75-8.

\section{Appendix}

SPECIFIC QUESTION:

A child arrives as an emergency in casualty not breathing and pulseless. He has been adequately intubated and ventilated, given $100 \%$ oxygen, and intravenous access has been rapidly secured. Effective basic life support is being administered. On your arrival what would your management be if the ECG revealed:*
(A) Asystole
(B) Ventricular fibrillation

GENERAL QUESTIONS:

1. Do you have MRCP?

2. Have you received in-house teaching on advanced life support in children?

3. Have you received APLS training?

4. If intravenous access had been impossible to attain within 90 seconds how would you have attained access?

5. If vascular access was not attained by three minutes how would you administer drugs?

${ }^{\star}$ Respondents were asked to reveal firstly their sequence of management and later to comment on specific areas they identified. 